Orthopäde $2017 \cdot 46: 893$

https://doi.org/10.1007/s00132-017-3480-9

Online publiziert: 27. September 2017

๑) Springer Medizin Verlag GmbH 2017

CrossMark

B. Rath $\cdot$ M. Tingart

Klinik für Orthopädie, Uniklinik RWTH Aachen, Aachen, Deutschland

\title{
Gelenkspezifische Verfahren zur Reparation hyalinen Knorpelgewebes
}

den sowie neuartige zell- und wachstumsfaktorbasierte Therapieansätze.

Die größte Erfahrung bei der Behandlung chondraler und osteochondraler Defekte besteht am Kniegelenk. Vor allem die Mikrofrakturierung und die autologe Knorpelzelltransplantation haben hier ihren Ursprung genommen.

Die Behandlung des Schulter-, Hüftund Sprunggelenkes ist aufgrund der anatomischen Voraussetzungen, der operativen Möglichkeiten sowie der Inzidenz und Art der Knorpelläsionen sehr spezifisch. Zum Beispiel führt ein symptomatisches CAM-Impingement am Hüftgelenk unbehandelt zu einer Knorpelläsion im Bereich des Acetabulums, die durch eine rechtzeitige Therapie weitestgehend vermieden werden kann. Arthroskopische Behandlungen des Hüftgelenkes sind auch heute noch vergleichsweise aufwendig und erfordern häufig den Einsatz von Spezialinstrumenten. Dennoch stellen sie eine minimalinvasive Alternative zur operativen Hüftluxation dar. Des Weiteren kann der Knorpelersatz an schwierig zu erreichenden Lokalisationen, wie $z$. B. dem Sprunggelenk zunehmend arthroskopisch assistiert über eine Miniarthrotomie durchgeführt werden, sodass hier eine Innenknöchelosteotomie oft vermieden werden kann.

Das Ziel dieses Themenheftes istes, die gelenkspezifischen Details und Besonderheiten der operativen Versorgungs- möglichkeiten von Knorpeldefekten an Schulter-, Hüft-, Knie- und Sprunggelenk darzustellen und einen Überblick über neue Therapieansätze zu geben.

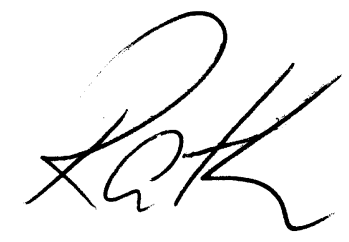

B. Rath

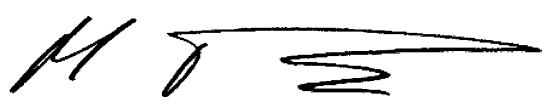

M. Tingart

\section{Korrespondenzadresse}

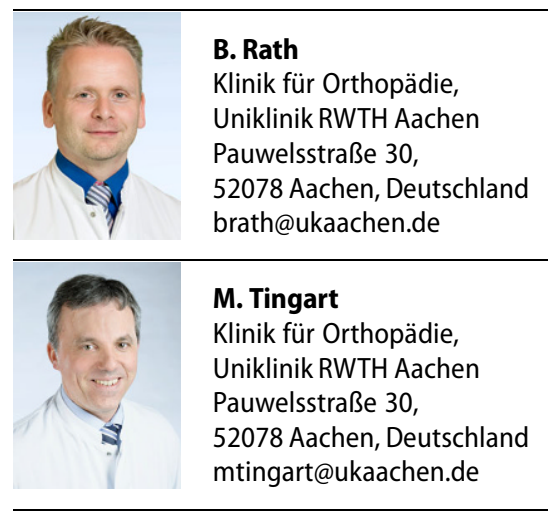

Interessenkonflikt. B. Rath und M. Tingart geben an, dass kein Interessenkonflikt besteht.
Die aktuellen Therapieformen beinhalten zellfreie und zellbasierte Metho- 